\title{
Change Detection Algorithms Analysis over Radar Images Using a Generating Function
}

\author{
Leonid G. Dorosinsky ${ }^{1, \text { a) }}$, Nina S. Vinogradova ${ }^{1, b)}$ \\ ${ }^{1}$ Yeltsin Ural Federal University, Yekaterinburg, Russia \\ a) Corresponding author: n.s.vinogradova@urfu.ru \\ b) 1.g.dorosinsky@urfu.ru
}

\begin{abstract}
While solving different problems of space monitoring, the problem of determining the presence or absence of the small changes of the reflecting surface arises during the observation session. For reliable detection of such a situation, it is recommended to use a constellation of small satellites that form statistically independent radar images. In the paper, we investigate the algorithm for classification of the signals reflected from two surfaces with different values of scattering crosssection on the set of radar images. The analytical method and the statistical simulation method are investigated, and the behavior of probabilities is examined when the magnitude of changes on the surface are varies. The recommendations are given on the choice of the number of satellites for various characteristics of reflective surfaces.
\end{abstract}

Keywords: radar remote sensing, scattering cross section, statistical simulation, change detection.

\section{INTRODUCTION}

The problem of detecting the underlying surface regions having the differences from the background the scattering cross-section is one of the most important in modern sense remote data tasks. An example is forecasting of ice conditions, when the wet areas should be detected at a uniform ice surface, monitoring the unauthorized forest loggings, observation of crop condition, etc. An effective solution (to the problem based on the results of processing a single observation data) is often impossible. Therefore, a method applying the usage of radar systems implemented by the small satellite constellations seems to be promising. The paper is devoted to analysis of the statistical simulation method for the study of such systems, as well as, the limit assessment of its applicability.

\section{DEVELOPMENT OF AN ALGORITHM CLASSIFICATION}

The signal at the primary processor output of the onboard radar can be represented as a sequence of $N$ random samples, all of which have an exponential distribution with a variation $\sigma_{i}^{2}$. Its value is proportional to the scattering cross-section. We need to classify two radar images with a minimal error

- all the samples of the first radar image data are statistically homogeneous and each of them has the same variance $\sigma_{0}^{2}$ (hypothesis $H_{0}$ );

- the data of the second radar image differs from the first one by presence of the "gap" array consisting of $\delta(\delta<N)$ samples with another variation. (Fig. 1a) (hypothesis $H_{1}$ ).

The classification error probability $P$ is equal to the sum of the probability of a missing error probability $P_{M}$ (when the hypothesis $H_{1}$ is valid, but the situation corresponds to the hypothesis $H_{0}$ ) and a false alarm error probability $P_{F}$ (when the hypothesis $H_{0}$ is valid, but the situation corresponds to the hypothesis $H_{1}$ ):

International Conference of Numerical Analysis and Applied Mathematics ICNAAM 2019

AIP Conf. Proc. 2293, 140021-1-140021-5; https://doi.org/10.1063/5.0027277

Published by AIP Publishing. 978-0-7354-4025-8/\$30.00 


$$
\begin{gathered}
P=\frac{1}{2} P_{M}+\frac{1}{2} P_{F}, \\
P_{M}=\int_{Z_{0}} p_{z \mid H_{1}}\left(\boldsymbol{Z} \mid H_{1}\right) d \boldsymbol{Z}, \\
P_{F}=\int_{Z_{1}} p_{z \mid H_{0}}\left(\boldsymbol{Z} \mid H_{0}\right) d \boldsymbol{Z},
\end{gathered}
$$

where $Z$ is the $N$-dimension observation vector, $p$ is the probability density distribution of the $Z$.

The most favorable value of the threshold $\eta$ can be found as the one when the probability $P$ takes the smallest value of all the possible ones. The threshold selection algorithm is shown in the Fig. 1b.

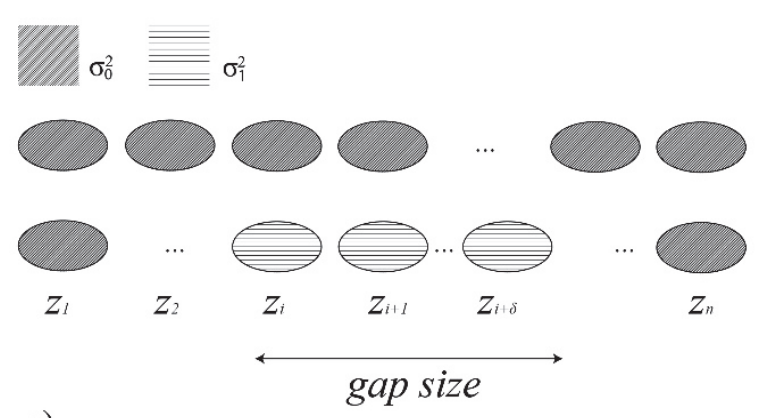

a)

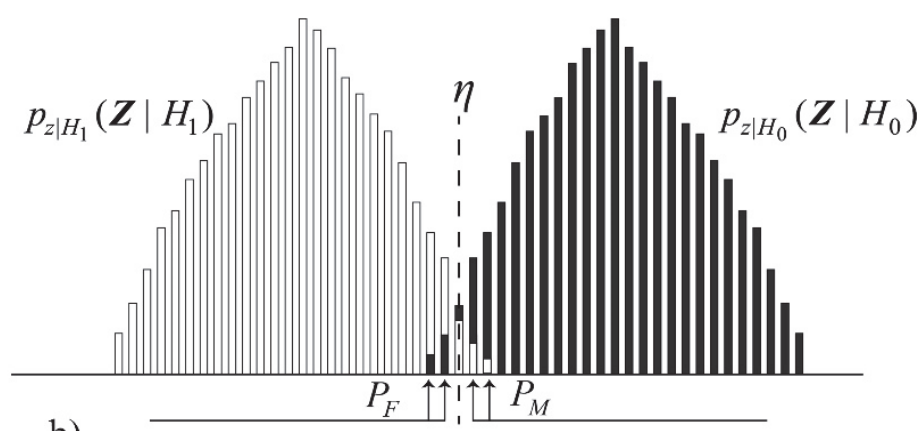

b)

FIGURE 1. a) The input data scheme for the simulation of variance gap;

b) algorithm of choosing the optimal threshold value using statistical simulation

Despite the fact that the proposed algorithm is quite clear, it is necessary to make sure in its correctness by comparing with the results obtained using the traditional analytical expressions [2]. The case when both surfaces are homogeneous is considered in detail in [1]. In the case when the second surface is not homogeneous, it is advisable to use the generating function according to the hypothesis $H_{0}$, which can be represented as follows:

$$
\begin{aligned}
& \Phi_{\Lambda(\boldsymbol{Z}) \mid H_{0}}(s)=\int_{-\infty}^{\infty} e^{s L} p_{l \mid H_{0}}\left(L \mid H_{0}\right) d L=e^{\mu(s)}, \\
& \mu(s)=\ln \int_{-\infty}^{\infty}\left\{p_{z \mid H_{1}}\left(\boldsymbol{Z} \mid H_{1}\right)\right\}^{s}\left\{p_{z \mid H_{0}}\left(\boldsymbol{Z} \mid H_{0}\right)\right\}^{1-s} d \boldsymbol{Z},
\end{aligned}
$$

where $s$ is the real variable defined on the set from 0 to $1, \mu(s)$ is the characteristic function with a mean value of the neighbourhood of the threshold $\eta$. In a situation where surfaces may be represented as $\sigma_{0}^{2}=\left\{\sigma_{01}^{2}=\ldots=\sigma_{0 i}^{2}=\ldots=\sigma_{0 N}^{2}\right\}$ and $\sigma_{1}^{2}=\left\{\sigma_{01}^{2}=\ldots \neq \sigma_{1 N-\delta}^{2}=\ldots=\sigma_{1 \delta}^{2} \neq \ldots=\sigma_{0 N}^{2}\right\}, \sigma_{0 i}^{2} \neq \sigma_{1 i}^{2}$, the $\mu(s)$ turns into

$$
\mu(s)=\sum_{1}^{N} \ln \left(\frac{q_{i}^{1-s}}{1+(1-s)\left(q_{i}-1\right)}\right)
$$

where $\boldsymbol{q}=1+\sigma_{0}^{2} / \sigma_{1}^{2}$.

Values of the missing and false alarm errors' probabilities are

$$
\begin{gathered}
P_{M}=\frac{1}{2} \exp \left[\mu(\eta)+\frac{\left(1-s_{m}\right)^{2}}{2} \ddot{\mu}(\eta)\right] \operatorname{erfc_{*}}\left(\left(1-s_{m}\right) \sqrt{\ddot{\mu}(\eta)}\right), \\
P_{F}=\frac{1}{2} \exp \left[\mu(\eta)+\frac{s_{m}^{2}}{2} \ddot{\mu}(\eta)\right] \operatorname{erf} c_{*}\left(s_{m} \sqrt{\ddot{\mu}(\eta)}\right),
\end{gathered}
$$

while the threshold value $\eta$ is found from the equation $\dot{\mu}(s)=0$. 


\section{ANALYSIS OF THE STATISTICAL SIMULATION METHOD APPLICABILITY}

Firstly, let us find the conditions, under which the results obtained using the computer simulation correspond to the calculated ones (4). Let us perform the following experiment: plot the classification error probability versus the variance ratio $\sigma_{0}^{2} / \sigma_{1}^{2}$ using (4), as well as, the method based on the statistical simulation for the different number of samples. The gap size $\delta$ is assumed to be fixed and equal to $50 \%$ of the total sample number, the number of realizations is 5000. The results are shown in Fig. 2a. As it is shown, when the number of samples is about 500, the simulation results almost match with the theoretical expression (4), the difference is not more than $1.5 \%$. With a smaller number of samples, the classification error probability obtained using expression (4) is underestimated in comparison with the probability value in simulation with 500 samples. This is due to the deviation from the normality of the initial distributions. When the variance ratio is 0.4 , the number of samples is 196 and the gap size is 98, and expression (4) gives an error probability of $0.06 \%$, while simulation gives the one of $7.88 \%$ (Fig. 2b). Similarly, for the number of samples equals to 100,324 , and 484 (with the fixed gap size of $50 \%$ ), the error probabilities are $15.75 \%, 3.74 \%$, and $1.2 \%$, respectively. With a smaller number of samples, the assumption that the initial distributions are normal does not hold; therefore, (4) is less accurate.

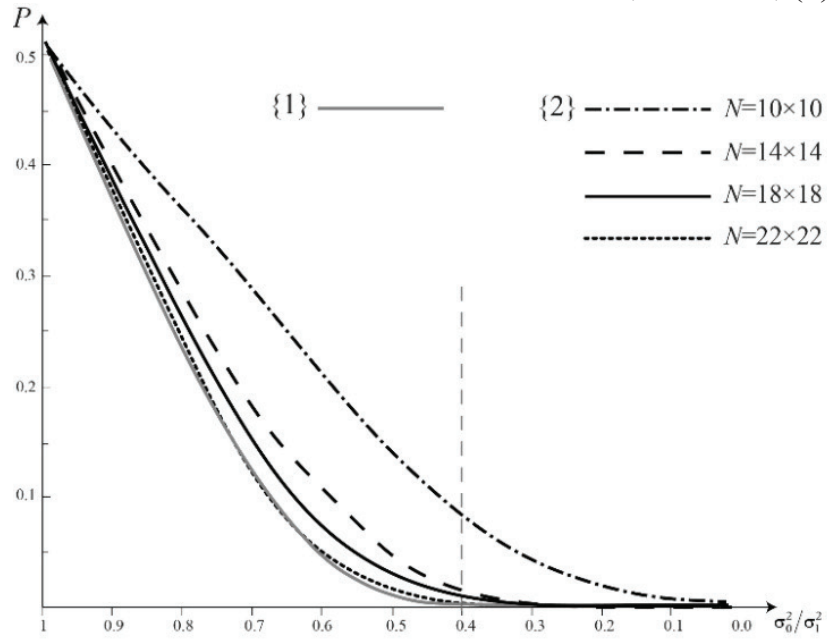

a)

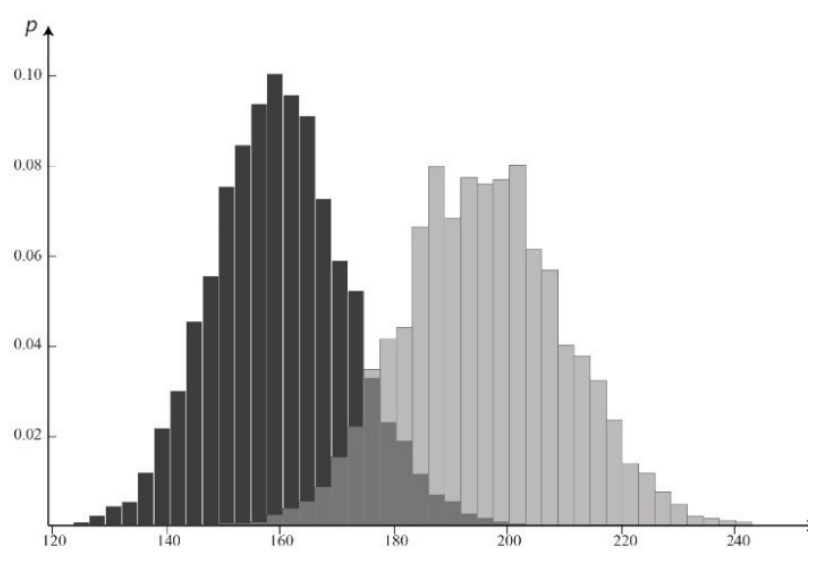

b)

FIGURE 2. a) The dependence of the error probability on the variance ratio (for the fixed gap size $\delta$ at $50 \%$ ); the gray line $\{1\}$ corresponds to the formula calculation, the black ones $\{2\}$ - to the statistical simulation with different sample numbers;

b) The histogram distributions of sufficient statistics for $N=196$ and $\delta=98$ (variance is equal to 0.4 ).

The dependence of the error probability on the gap size for different variance ratios is shown in Fig. 3. 


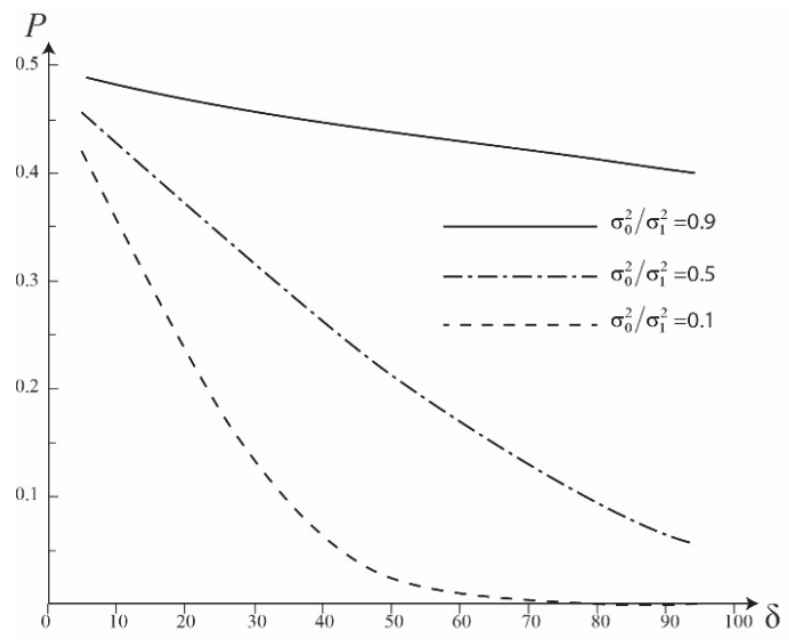

a)

FIGURE 3. The dependence of the error probability on the size gap with anomalous dispersion for a) 100 samples; b) 256 samples.

It should be noted that for small values of the variance ratio, there is some threshold gap size, at which the error probability takes a zero value. Whereas, for variance ratios higher than 0.5 , the error is non-zero under any conditions, and with a decrease in the sample numbers this effect becomes stronger. The threshold values of the gap sizes for a different sample number are given in Table 1.

TABLE 1. The threshold values of gap size, for which the error probability is equal to zero.

\begin{tabular}{rrrrrrrrrrrrrr|r|r|r|}
\hline$\sigma_{0}^{2} / \sigma_{1}^{2}$ & 0.05 & 0.1 & 0.15 & 0.2 & 0.25 & 0.3 & 0.35 & 0.4 & 0.45 & 0.5 & 0.55 \\
\hline$N=100$ & 73 & 85 & 91 & 97 & \multicolumn{1}{c}{-} & \multicolumn{1}{c}{-} & \multicolumn{1}{c}{-} & \multicolumn{1}{c}{-} & \multicolumn{1}{c}{-} & - \\
\hline$N=144$ & 93 & 106 & 119 & 128 & 139 & 143 & - & - & - & - & - \\
\hline$N=256$ & 114 & 155 & 167 & 173 & 184 & 223 & 242 & 253 & - & - & - \\
\hline$N=324$ & 153 & 169 & 196 & 201 & 237 & 261 & 279 & 295 & 313 & 322 & - \\
\hline
\end{tabular}

In particular, when the number of samples is 144 and the variance ratio is 0.1 , it is enough for the gap size to be at least 62 samples for a zero-classification error probability; whereas, for a variance ratio of 0.25 , the corresponding parameter should be at least 95 samples. With a variance ratio of 0.3 , the classification error probability is different from zero for any gap sizes of the anomalous scattering cross-section. For the close scattering cross-section values of the classified surfaces, the single observation is not enough, and to obtain sufficient efficiency, multiple several sources should be integrated (for example, $L$ satellites).

\section{ANALYSIS OF CLASSIFICATION EFFICIENCY}

In the case of independence of each observation, the probability of making the right decision of the system as a whole $P_{\varepsilon}$ and the probability of an individual means of observation $P$ are related as:

$$
P_{\varepsilon}=1-P^{L}
$$

For example, to obtain the final probability of correct recognition equal to 0.9 , it is sufficient to use eleven satellites with the correct recognition probability of 0.2 or three satellites with the correct recognition probability of 0.5 . Table 2 shows the necessary quantities of observation means to obtain a given final probability for a number of variance ratios. The number of samples is fixed and set at 100 . 
TABLE 2. The necessary quantities of observation means to obtain the final classification probability equaled to $99.9 \%, 99 \%$, and

\begin{tabular}{|c|c|c|c|c|c|c|c|c|c|c|c|c|c|c|c|c|}
\hline & \multicolumn{4}{|c|}{$P \varepsilon=99.9 \%$} & \multicolumn{4}{|c|}{$P \varepsilon=99 \%$} & \multicolumn{4}{|c|}{$P \varepsilon=95 \%$} & \multicolumn{4}{|c|}{$P \varepsilon=90 \%$} \\
\hline$\sigma_{0}^{2} / \sigma_{1}^{2}$ & $\delta=10 \%$ & $25 \%$ & $50 \%$ & $75 \%$ & $10 \%$ & $25 \%$ & $50 \%$ & $75 \%$ & $10 \%$ & $25 \%$ & $50 \%$ & $75 \%$ & $10 \%$ & $25 \%$ & $50 \%$ & $75 \%$ \\
\hline 0.3 & 8 & 6 & 4 & 2 & 6 & 4 & 3 & 2 & 4 & 3 & 2 & 1 & 3 & 2 & 2 & 1 \\
\hline 0.4 & 8 & 7 & 4 & 3 & 6 & 5 & 3 & 2 & 4 & 3 & 2 & 2 & 3 & 3 & 2 & 1 \\
\hline 0.5 & 8 & 7 & 5 & 4 & 6 & 5 & 3 & 3 & 4 & 3 & 2 & 2 & 4 & 3 & 2 & 2 \\
\hline 0.6 & 8 & 8 & 6 & 5 & 6 & 5 & 4 & 4 & 4 & 4 & 3 & 3 & 4 & 3 & 2 & 2 \\
\hline 0.7 & 10 & 8 & 7 & 5 & 7 & 6 & 5 & 4 & 5 & 4 & 3 & 3 & 4 & 3 & 3 & 2 \\
\hline 0.8 & 10 & 9 & 8 & 7 & 7 & 6 & 5 & 5 & 5 & 4 & 4 & 3 & 4 & 4 & 3 & 3 \\
\hline 0.9 & 10 & 10 & 9 & 8 & 7 & 7 & 6 & 6 & 5 & 5 & 4 & 4 & 4 & 4 & 4 & 3 \\
\hline
\end{tabular}

It is noted from Table 2, obtaining reliable results using a single observation tool is possible only in the case of both the large gap size and its significant "depth". The probability of correct classification does not generally exceed $90 \%$. To obtain the correct classification probability of $99 \%$ and more, it is recommended to use at least three spacecrafts. The results relate to a model based on 100 samples during an increase of its, the required number of observation means decrease, since both factors lead to an increase in the observation statistics, and, as a result, to the reduce of classification error.

\section{CONCLUSION}

The method of the statistical simulation was used for studying the possibility of detecting small changes on a uniform background of the radar image. The simulation results were compared with the analytical expressions, and it was shown that with the number of samples less than 500, the simulation gives more accurate results due to the failure of fulfitting the assumption of normality of the distributions formed by the analytical expressions. It is shown that the classification error probability decreases both with a decrease of the variance ratio of classified areas over radar images and with an increase in the gap size relative to the total number of samples. The recommendations on the choice of the required number of observation means for observing surfaces with different variance ratios are developed.

\section{REFERENCES}

1. Dorosinsky L. G., Vinogradova N. S. The investigation of change detection algorithms for radar images using the multiposition space monitoring system. Ural Radio Engineering Journal.; vol 3(2):132-156. (2019) DOI: 10.15826/urej.2019.3.2.002

2. Van Tris G. Theory of detection, estimates and modulation. (in russuan). Sovetskoe radio, Russia, 344 p (1971). 